P. MAHNKE

H.H. KLINGENBERG ${ }^{1}$

A. FIX ${ }^{2}$

M. WIRTH ${ }^{2}$

\title{
Dependency of injection seeding and spectral purity of a single resonant KTP optical parametric oscillator on the phase matching condition
}

\author{
${ }^{1}$ Institut für Technische Physik, Deutsches Zentrum für Luft- und Raumfahrt (DLR), Pfaffenwaldring 38-40, \\ 70569 Stuttgart, Germany \\ 2 Institut für Physik der Atmosphäre, Deutsches Zentrum für Luft- und Raumfahrt (DLR), Oberpfaffenhofen, \\ 82234 Wessling, Germany
}

\begin{abstract}
Received: 12 December 2006/Revised version: 16 July 2007
Published online: 18 August 2007 • (C) Springer-Verlag 2007

ABSTRACT For spectroscopic and remote sensing applications injection seeded optical parametric oscillators (OPOs) are well established. In this paper we study the dependencies of signal resonant injection seeding of an OPO on its resonator length, phase matching angle and pump power in detail. The quality of the seeding process is assessed by stabilising the seed laser on a molecular absorption line of water vapour and using a water vapour absorption cell as a narrow bandwidth filter for the injection seeded radiation. A reduction of the acceptance of injection seeding is observed with increasing pump power. For small phase mismatch injection seeding with a spectral purity of $99.7 \%$ was observed at 13 -fold OPO threshold. A signal pulse energy of $38 \mathrm{~mJ}$ with $50 \%$ pump depletion was achieved with a beam parameter $\mathrm{M}^{2}$ of about 6 .
\end{abstract}

PACS 42.65.Yj; 42.79.Nv; 42.79.Qx

\section{Introduction}

Injection seeded optical parametric oscillators are versatile frequency converters for spectroscopic applications and remote sensing. For air- and space-borne differential absorption lidar (DIAL) they are well suited transmitter systems because the excess energy in the frequency conversion process generates radiation and usually no heat. The compactness of OPO resonators enables robust transmitter designs. The stringent operating requirements for these applications are a high pulse energy, a high conversion efficiency, a high spectral purity, and a low beam divergence. These features are not easily obtainable by linearly configured OPOs, because they need a very high degree of optical isolation for injection seeded operation. However, in a ring configuration these parameters can be realized. A transmitter consisting of a frequency doubled injection seeded Nd:YAG pump laser and an OPO as a frequency converter switchable seeded/unseeded ring $\mathrm{OPO}$, was used for $\mathrm{H}_{2} \mathrm{O}$-DIAL [1].

In the following we describe the experimental setup and details of the performed measurements. To evaluate the design parameters for the OPO, such as crystal length and crystal

Fax:+49-711-6862-715, E-mail: peter.mahnke@dlr.de angle tolerance, we chose for injection seeding one wavelength to study the dependence of its spectral properties on the detuning of the phase matching condition $\Delta k$ and resonator length $l$. For the measurement an injection seeded single resonant ring OPO was set up, and investigated for pump energies exceeding the threshold by more than eight times. The injection seeding behaviour of the OPO was studied using a narrow bandwidth filter for the injection seeded wavelength. This will be summarised in Sect. 3 .

As an additional new feature, we believe we have observed second order injection seeding of the OPO resonator. At a pump power level where the OPO operates at a factor of 2.5 above the threshold we found injection seeding between adjacent longitudinal modes of the OPO resonator. This is described in Sect. 4.

2

\section{Experimental}

The setup consists of a single longitudinal mode frequency doubled Q-switched Nd:YAG laser with a pulse length of $7 \mathrm{~ns}$, a repetition rate of $100 \mathrm{~Hz}, 18$ Watt average power and a beam diameter of $4.8 \mathrm{~mm}$. This laser pumps a walk-off compensated injection seeded dual crystal single resonant ring OPO with idler dumping between the crystals as shown in Fig. 1. The $532 \mathrm{~nm}$ light pumps the KTP crystals entering the resonator passing through mirror M1 and leaving the resonator through mirror M4. Mirror M2 acts as the output coupler of the $935 \mathrm{~nm}$ signal resonant OPO. To obtain injection seeding, the resonator length can be controlled by moving mirror M3 with a piezo electric transducer. The $1234 \mathrm{~nm}$ idler light is coupled out behind each crystal to prevent a fixed phase relationship of signal, idler and pump light at the next crystal, when the OPO is injection seeded. This idler dumping also prevents back conversion in the second crystal as well as resonance effects due to the interaction between idler, signal and pump phases. The two KTP crystals were type II $(\mathrm{o} \rightarrow \mathrm{oe})$ phase matched for $935 \mathrm{~nm}$, cut under an angle $\theta=70.7^{\circ}, \phi=0^{\circ}$ with an aperture of $8 \times 8 \mathrm{~mm}^{2}$, and $10 \mathrm{~mm}$ length. The KTP crystals were anti-reflection coated for the pump, signal and idler radiation. To adjust the phase matching angle $\theta$ both crystals were individually mounted on a rotation stage. For injection seeding, a fibre coupled external cavity diode laser with an output power of $2 \mathrm{~mW}$ was used. The external cavity diode laser was stabilised onto a strong ab- 


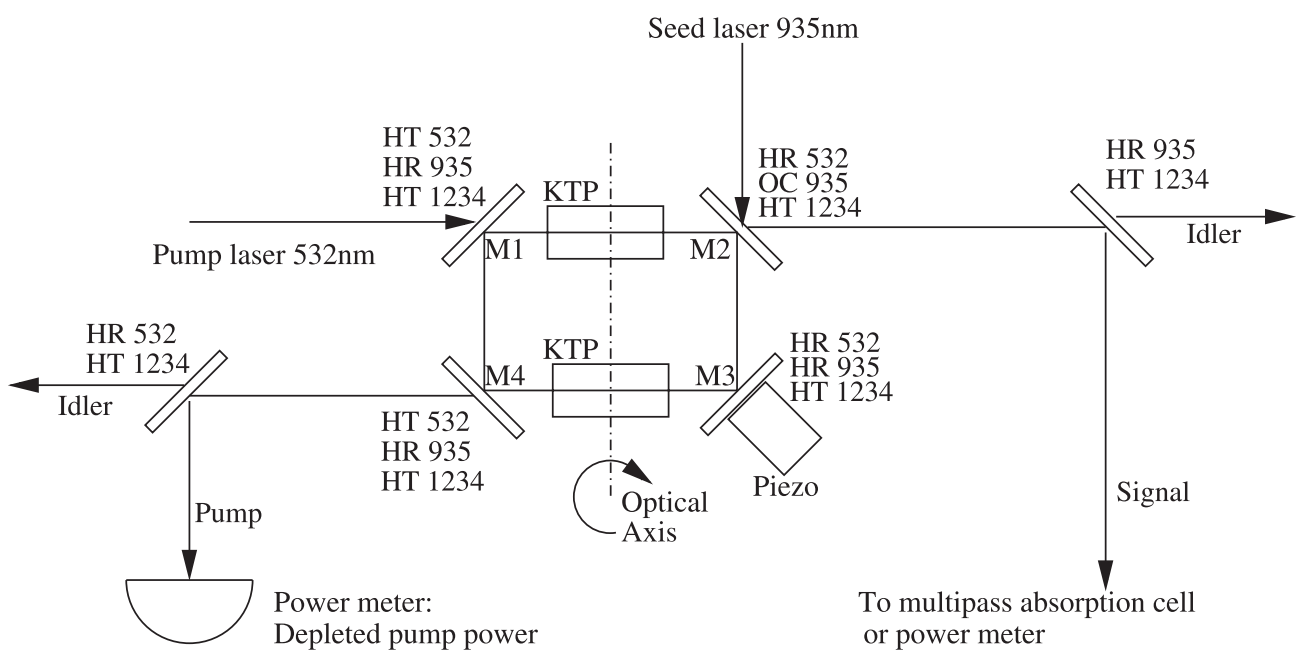

FIGURE 1 Experimental setup of the walk-off compensated dual crystal injection seeded OPO. HR: highly reflective coating, HT: highly transmitting coating, OC: output coupler

sorption line of water vapour at $935.45 \mathrm{~nm}$. The optical length of the resonator was approximately $13 \mathrm{~cm}$ which corresponds to a free spectral range of $2.3 \mathrm{GHz}$.

The signal radiation was analysed either by a power meter or a $100 \mathrm{~m}$ long multi-pass absorption cell (new focus model 5612). This absorption cell is used to determine the spectral purity of the pulsed injection seeded optical source, which is defined as the ratio of the energy present in the injection seeded mode $I_{\text {seeded }}$ and the total energy of the pulse $I_{\text {total }}$. The power present in the injection seeded longitudinal mode is determined indirectly by measuring the power present in the unseeded longitudinal modes. For this purpose the absorption cell was filled with $15-20 \mathrm{hPa}$ of water vapour as a narrow bandwidth filter for the injection seeded mode. The seed laser was stabilised onto a strong water vapour absorption line at $935.45 \mathrm{~nm}$, where the residual transmission ratio $T_{\text {Cell }}$ through the cell is smaller than $10^{-5}$. The absorption line width was approximately $1.3 \mathrm{GHz}$ [4]. The spectral purity of our shown measurements is then given by:

$\frac{I_{\text {seeded }}}{I_{\text {total }}}=1-\frac{I_{\text {total }}-I_{\text {seeded }}}{I_{\text {total }}} \approx 1-T_{\text {Cell }}$.

The phase mismatch $\Delta k$ of the injection seeded mode was measured using an optical spectrum analyser with a full width half maximum resolution of $0.03 \mathrm{~nm}$. This was done by measuring the wavelength difference $\Delta \lambda$ between the maximum of the OPOs free running spectrum and the maximum of the spectrum of the injection seeded mode.

\section{Results}

Firstly, we discuss the general performance of the OPO. The pump power dependence of the conversion efficiency of the OPO was measured for different output couplers. Only a slight absorption in the crystals was detected. The injection seeding stability was then measured by using the multi pass absorption cell to determine the degree of single mode operation, which is the spectral purity of the OPO. For this purpose the injection seed laser was tuned to a molecular absorption of water vapour as described before. Single mode operation was demonstrated at a pump power exceeding the OPOs threshold by 13 times. This was shown with nearly per- fect phase matching. A decrease of the spectral purity of the OPO was observed due to temperature changes in the crystal during the measurement.

Secondly, we investigated the OPOs injection seeding stability for different pump energies and crystal angles. The resonator length of the OPO was scanned with the piezo element for fixed pump powers and crystal angles. This was performed to explore the possibility of accessing multiple wavelengths with high spectral purity using only one optical parametric oscillator. The questions arising from this are: What phasemismatch is allowed for the injection seeded mode? Is it necessary to tilt the crystal? What happens at high pump energies exceeding the OPOs gain saturation? How does the resonators finesse influence injection seeding?

\subsection{Performance of the OPO}

To characterise the performance of the OPO we measured the conversion efficiency in terms of pump depletion.

Figure 2 shows the measured pump depletion of the unseeded OPO for various output couplers. The data were obtained by calculating the quotient of the measured pump energy, which is transmitted through the OPO setup while the OPO is operating, and the pump energy, which is transmitted through the OPO with the OPO resonator blocked between mirror M4 and M1. The pump depletion is directly connected to the parametric process because the reflection losses of the mirrors cancel out. Additionally, the conversion efficiency of the OPO is characterised by this method.

In Fig. 2a, the pump depletion is plotted as function of the number of times the pump power exceeds OPO threshold for various reflectivities of the output coupler. The plot shows well that no dependence of the conversion efficiency on the reflectivity of the output coupler. The conversion efficiency of an OPO is limited by the maximum parametric efficiency which can be reached in a cw pumped OPO with a gaussian beam shape, which is $73 \%$ at a pump power approximately seven times above the OPO threshold [10], and the maximum parametric efficiency which can be reached by the temporal pulse shape of an $\mathrm{sech}^{2}$. The resulting maximum total conversion efficiency of an optical parametric oscillator is therefore 


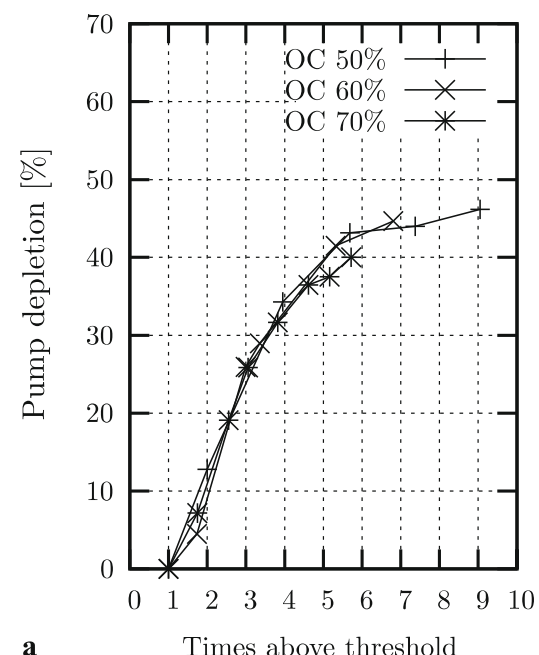

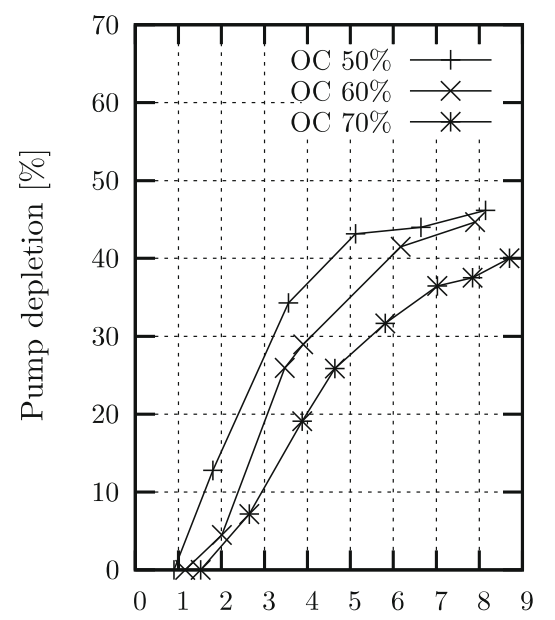

b Pump power (behind the resonator) $[\mathrm{W}]$

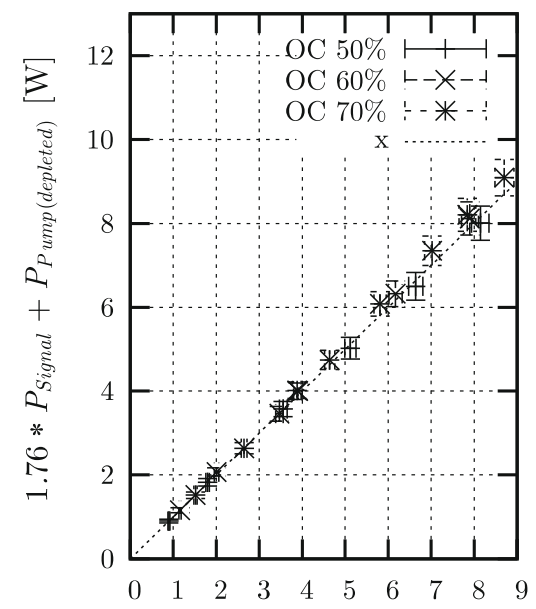

c Pump power (behind the resonator) $[\mathrm{W}]$

FIGURE 2 Pump power depletion (conversion efficiency) of the dual crystal KTP OPO for different output couplers, with 50\%, 60\%, and 70\% transmission, respectively. From left to right: (a) Conversion efficiency plotted versus OPO threshold; (b) conversion efficiency versus pump power; (c) power balance of the OPO

approximately $50 \%$, which is in good agreement with our measured data.

Figure $2 \mathrm{~b}$ depicts the dependence of the pump depletion on the pump power for different output couplers. An increase of the OPOs threshold with the increase of the output coupling is observed. Because the conversion efficiency of the OPO is dependent on the number of times the pump power exceeds the OPO threshold, the degree of output coupling can be used to choose the favourable working point of the optical parametric oscillator. For a stable injection seeded operation with a high spectral purity a working point around $5-6$ times above the OPOs threshold seems favourable (see Sect. 3.3).

Finally, to verify the measured pump depletion with the measured signal power we calculated the pump balance of the OPO, which is depicted in Fig. 2c. Both, the measured residual $532 \mathrm{~nm}$ pump power and the $935 \mathrm{~nm}$ OPO signal power were used to calculate the pump power without the parametric process. This was calculated by summing the residual pump power, the signal power and the idler power. The power of the idler radiation was not measured, since it was dumped at two mirrors. The parametric process requires for each signal photon, one idler photon and therefore, the $1234 \mathrm{~nm}$ idler power was calculated by taking the ratio of $\omega_{\text {idler }} / \omega_{\text {signal }}=0.76$ of the signal power. This calculated pump power is plotted versus the undepleted pump power, which was measured by blocking the parametric process. An almost perfect correlation between the undepleted pump power and the calculated pump power was observed. Only a small amount of power was lost by absorption in the nonlinear process.

Injection seeding of an OPO increases the maximum efficiency. This is reached by reducing the pulse build up time of the parametric oscillation, yielding an additional reduction of the OPO threshold. In Fig. 3 these two processes are illustrated. The lower solid curve is a polynomial fit of the measured data points. Furthermore, to determine the decrease of the OPOs threshold and the increase in efficiency by injection seeding, we measured the increase of power by injection seeding the OPO for $\Delta k=0$ at two arbitrary power levels (at $2.5 \mathrm{~W}$ 2 times and at $4.5 \mathrm{~W} 1.4$ times). Using this increase, indicated

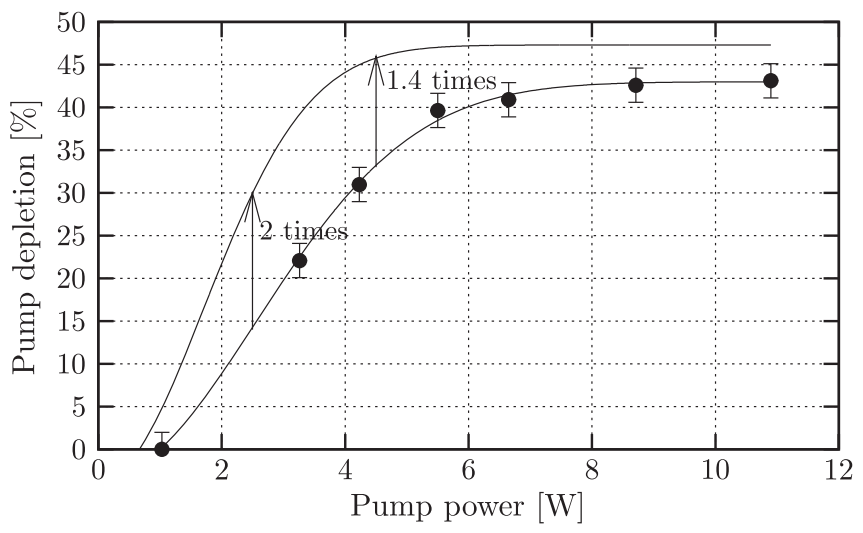

FIGURE 3 Pump depletion curve of the OPO: The dots with error bars indicate the measured power curve of the unseeded OPO. The lower solid line is a fit curve of the measured power curve. The upper solid line shows the estimated power curve for the injection seeded OPO for $\Delta k=0$

by the two arrows in the figure, an estimated power curve was calculated by scaling the fit curve in the pump power direction and pump depletion direction, depicted in the upper solid curve. Since, with increasing pump energy, thermal effects on crystals and resonator length become noticeable and therefore the measurement of the pump depletion in seeded operation requires an exact adjustment of the crystal angle and resonator length for each measurement point, while keeping all other parameters constant. For simplicity, we did this for two measurement points and scaled the curve accordingly.

\section{2}

\section{Spectral purity measurement}

In a long term measurement, shown in Fig. 4, a spectral purity of better than $99.7 \%$ was achieved at maximum pump energy and a signal energy of $38 \mathrm{~mJ}$. This was achieved by stabilising the resonator length on the lowest absorption cell transmission. The noise in the transmission cell signal is partly due to resonator length dithering of the stabilisation. The higher cell transmission at the end of the measurement is due to a small thermal change of the crys- 


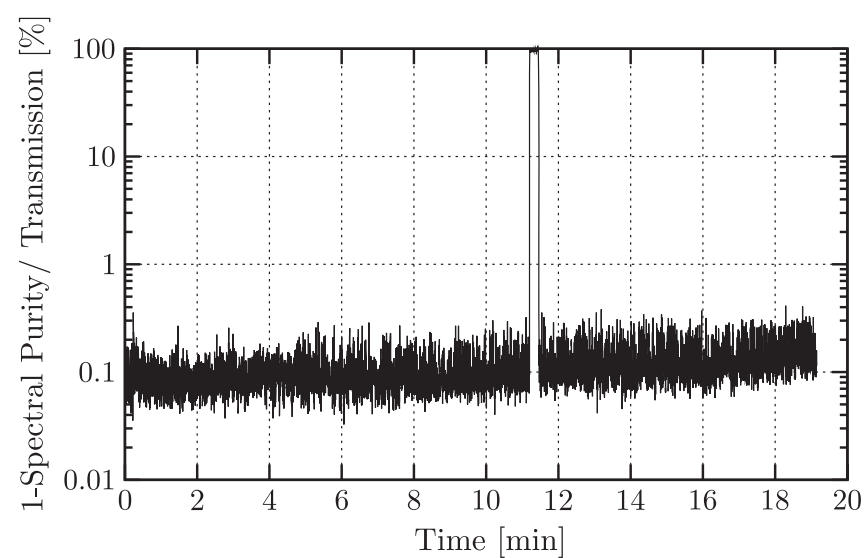

FIGURE 4 Long term spectral purity measurement $(20 \mathrm{~min}$ ) for the maximum pump power. In the 11th minute the seed beam was blocked to obtain a reference transmission

tal which results in a small phase mismatch of the injection seeded mode. At this power level we achieved an optical conversion efficiency of approximately $50 \%$ (signal and idler) which corresponds to $28 \%$ conversion efficiency for the signal radiation. The divergence of the OPO signal radiation was limited at this pump power by the angular acceptance of the KTP crystal to 1.7 mrad with a beam diameter similar to the beam diameter of the pump laser of approximately $5 \mathrm{~mm}$. The $M^{2}$ value under these conditions was measured to be 6 . For the measurement of the beam parameter a triggered CCD camera was used. The system was provided with a $6 \mu \mathrm{m}$ pitch and $\mathrm{a} \mathrm{f}=1000 \mathrm{~mm}$ lens. The measurement was performed in accordance with the ISO standard 11146.

\subsection{Dependence of spectral purity on phase mismatch $\Delta k$ and resonator length $l$}

To observe the dependence of the injection seeding efficiency on the phase mismatch, we measured the spectral purity for different phase matching angles while scanning the resonator length. A series of measurements was performed for different pump powers: $2.5,4.8$ and 10 times above the threshold, respectively. These measurements are depicted in Fig. 5 from left to right. The observed spectral width of the free running OPO during these measurements was $0.14 \mathrm{~nm}$ full width half maximum.

The asymmetries observed in the injection seeding behaviour with increased pump power, seen in the two right hand diagrams of Fig. 5, stem from the phase shift of the higher order transverse modes, which are not degenerate in this resonator. With increased pump power higher order transversal modes start to build up in the oscillator and the beam parameter $M^{2}$ increases. Because the resonator is short and composed of plane one inch mirrors the resonator has a rather large Fresnel number. The main loss mechanism for higher order transversal modes in the OPO is resulting from the lower gain of higher divergent modes in the nonlinear crystal caused by the limited angular acceptance of the crystals. This limits the divergence of the OPO signal beam to $1.7 \mathrm{mrad}$, which is observed at the maximum pump power resulting in a beam parameter $M^{2}$ between 6 and 10. For low average pump powers exceeding the threshold by 2.5 times the beam parameter $M^{2}$ of 2.5 was measured.

The decrease of the injection seeded OPOs spectral purity with increasing pump power is due to the increasing overall gain which results in two effects. At first, more longitudinal modes of the OPO are exceeding the threshold and start competing for the pump. And second, when the OPO is operated more than three times above the threshold, a significant back conversion of different signal and idler modes broaden the pump spectrum. As a consequence gain saturation is no longer negligible.

For low pump powers, where no significant back conversion occurs, no gain saturation was observed. In this case the OPOs behaviour can be described by modifying the model presented in [5] for more than two modes. However, in that
2.5 times above threshold

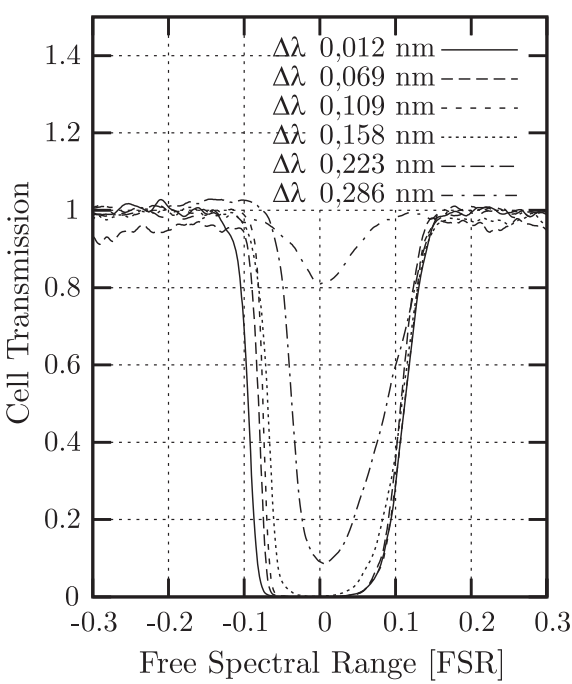

4.8 times above threshold

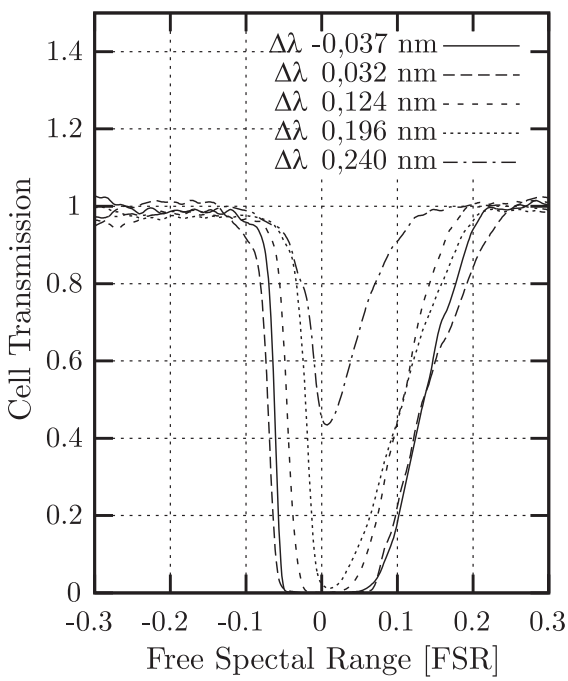

10 times above theshold

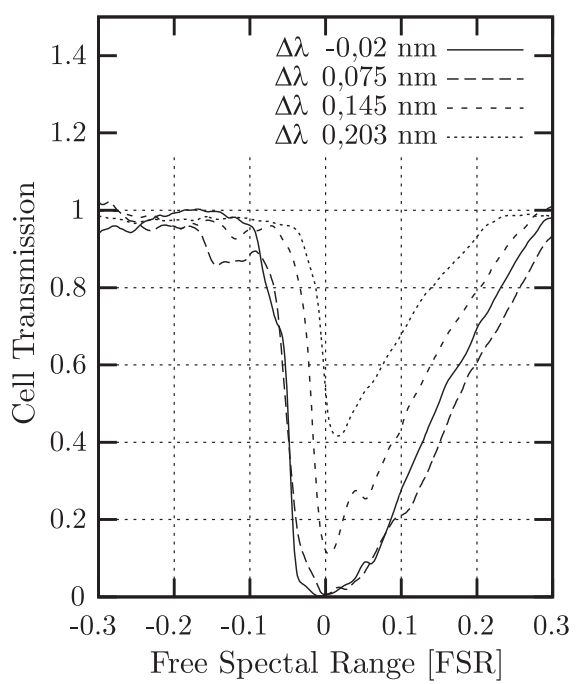

FIGURE 5 Cell transmission versus resonator length detuning in units of the resonators free spectral range for different crystal angles and pump powers. The different crystal angles are measured by the detuning $\Delta \lambda$ of the OPOs central wavelength with respect to the seed laser radiation. From left to right: OPO pumped 2.5 times above threshold, OPO pumped 4.8 times above threshold, and OPO pumped 10 times above threshold 
paper only two modes were considered with a linear dependence on the spectral property and the output power. The left plot of Fig. 6 shows the measured transition (relative power and relative cell transmission) from multimode to single mode operation when the resonator length is scanned. In this case we observed an exponential dependence. When the resonator length approaches the resonance of the seed laser the free running multiple modes get increasingly suppressed by the injection seeded mode. The mode suppression process of increasing seed energy was demonstrated in [7]. In our measurement a small hysteresis of the piezo is observed in the measured curves. The dotted curves are the measured relative power of the OPO during the scan, the power of the unseeded OPO is arbitrarily set to one. The measured relative power is fitted by an Airy function (upper solid curve) with two parameters given by the resonator finesse and the maximum power increase achieved by injection seeding. The power increase is proportional to the Airy function of the OPO resonator as long as no gain saturation occurs [5]. The measured cell transmission of the OPO, indicated by the dash dotted curves, is proportional to the exponent of the power, because all free running modes of the OPO get successively suppressed by the injection seeded mode $[6,7]$. The lower solid curve is the exponential Airy function which represents the measured cell transmission. Using these numerical fit curves, we can obtain an analytical description of the measured spectral purities for different phase mismatches $\Delta k$. This is obtained by scaling the Airy function of the power with $\operatorname{sinc}^{4}\left(\frac{\Delta k l}{2}\right)$ for the two crystals with length $l$ used in our OPO. The scaling of the signal power and the resulting cell transmission is presented in the right diagram of Fig. 5. The upper curves represent the relative power of the OPO and the lower curves the corresponding cell transmissions. For the scaling the same phase mismatches as in the measurement presented in the left diagram of Fig. 5 were chosen. The phase mismatch $\Delta k$ is given by:

$\Delta k(\Delta \omega)=\left(\frac{\mathrm{d} k}{\mathrm{~d} \omega}\left(\omega_{\text {Signal }}\right)-\frac{\mathrm{d} k}{\mathrm{~d} \omega}\left(\omega_{\text {Idler }}\right)\right) \Delta \omega$.

Taking the crystal data $(\mathrm{d} k / \mathrm{d} \omega)$ for the phase matching angle of the KTP [8] the resulting phase mismatch yields:
$\Delta k(\Delta \lambda) \approx \frac{0.2 \pi}{\lambda^{2}} \Delta \lambda$

The calculated cell transmission curves shown in the right hand side of Fig. 6, are in good qualitative agreement with the measured curves shown in the left diagram of Fig. 5.

The total phase matching range where injection seeding effects will be observed, can be estimated by measuring the power enhancement $g_{0}$ for $\Delta k=0$ and then calculating the point $\Delta k$ where unity gain is reached. The maximal power enhancement represented by the gain factor $g(\Delta k)$, which can be achieved by injection seeding, is given by:

$g(\Delta k)=g_{0} \operatorname{sinc}^{4}\left(\frac{\Delta k l}{2}\right) \approx g_{0}\left(1-\frac{\left(\frac{\Delta k l}{2}\right)^{2}}{6}\right)^{4}=1$.

Using this relationship and the power enhancement achievable for $\Delta k=0$, which is shown in Fig. 3 the $\Delta k$ range where injection seeding can be observed can be estimated. The estimations of the $\Delta \lambda(\Delta k)$ ranges where injection seeding can be observed for the pump powers used in the measurements of Fig. 5 result in: $\pm 0.27 \mathrm{~nm}$ for a pump power 2.5 times above the OPOs threshold, $\pm 0.19 \mathrm{~nm}$ for a pump power 4.8 times over the OPOs threshold, and $\pm 0.15 \mathrm{~nm}$ for a pump power 10 times over the OPOs threshold. These calculated ranges are smaller than the observed ranges, because the limiting saturation effects are not taken into account. Furthermore, the broadening of the longitudinal mode due to the non degenerate transverse modes is also not considered in this model.

The above relationship for the injection seeding acceptance bandwidth is nearly the relationship of the phase matching acceptance bandwidth. The main difference is that the injection seeding bandwidth is influenced by the power enhancement $g_{0}$ achieved at a certain pump power. Like all acceptance bandwidths of the parametric processes this acceptance bandwidth scales reciprocally with the crystal length. Therefore, a shorter crystal would result in a larger range where injection seeding would be observed, also in a higher threshold, and in a larger angle of acceptance. The greater angle of acceptance results in an increasing signal beam parameter $M^{2}$.
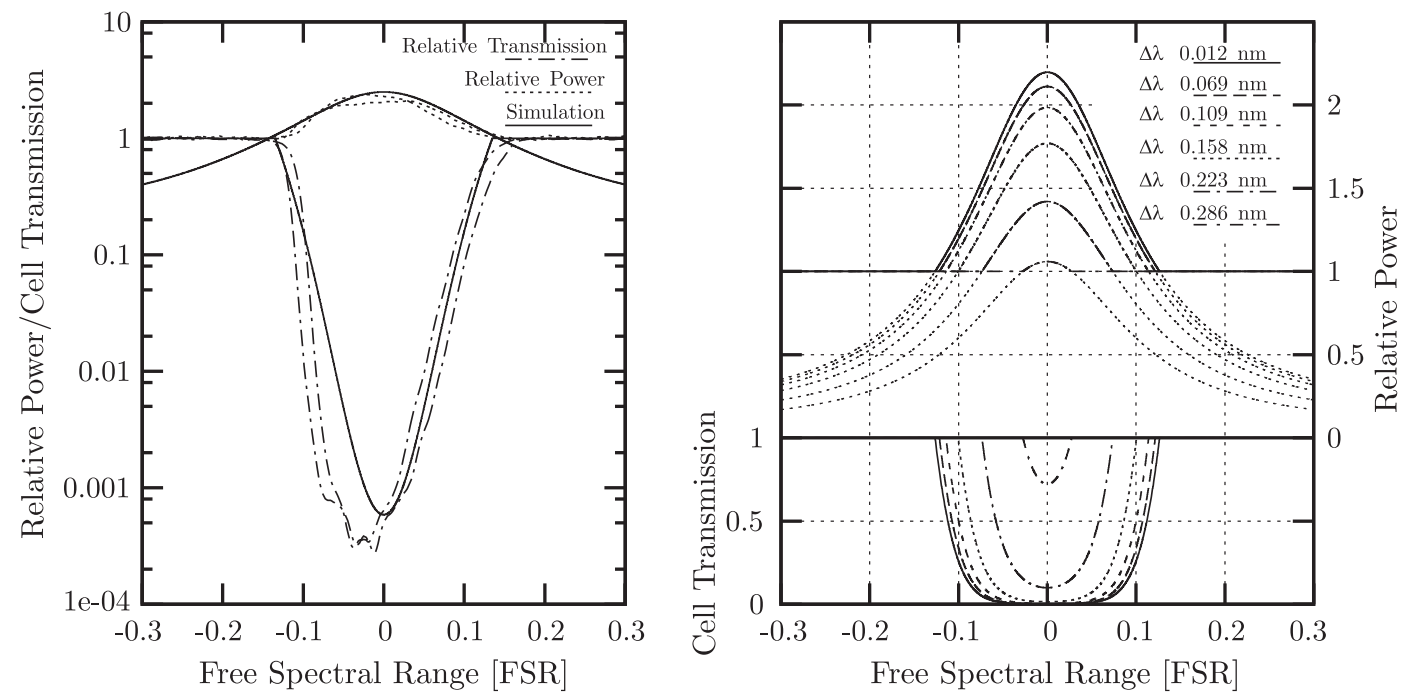

FIGURE 6 Left: Measured and simulated relative power and transmission curves of one back and forth scan of the resonator for $\Delta \lambda=0.012 \mathrm{~nm}$ and 2.5 times above threshold. Right: Simulated transmission and relative power curves for different $\Delta k(\Delta \lambda)$ similar to the curves shown in Fig. 5 for the OPO pumped 2.5 times above threshold 
To estimate the $\Delta k$ range where a specific spectral purity by injection seeding is achieved, unity in the given equation must be replaced by the measured power increase at this spectral purity. As an example, if we want a spectral purity better than $1 \%$ at a pump power 2.5 times above OPO threshold a signal power increase due to injection seeding of 2 is observed (see Fig. 6 on the left). This signal power increase can be achieved with a maximum phase-mismatch for the injection seeded mode, corresponding to a wavelength difference of $\Delta \lambda$ of 0.109 (see Fig. 6 on the right).

\section{$4 \quad$ Observation of second order injection seeding}

When the OPO resonator length is scanned and the OPO is operated at a low pump power and low phase mismatch for the injection seeded mode, a high power increase by injection seeding was observed at the main resonator resonances. Surprisingly, in the middle of two longitudinal modes of the resonator we observed injection seeding of the OPO with low spectral purity, which could correspond to a 2nd order resonance of the resonator (a double circulation of the light in the resonator). This finding is illustrated by the measurement shown in Fig. 7 at -0.5 and 0.5 free spectral ranges. The measurement was performed at a pump power exceeding the OPO threshold by approximately 2.5 times and at a phase mismatch $\Delta k$ near zero. The relative signal power of the OPO, upper curves, and the cell transmission, lower curves, are shown. The relative power and the cell transmission value of the unseeded optical parametric oscillator is arbitrarily set to one. The measured relative power increase observed at the main resonances was fitted with the Airy function of the resonator $\frac{1}{1-2 r \cos (\Delta \phi)+r^{2}}$ with a reflectivity $r=0.5$, and a maximum power increase by a factor of 2.5 . The measured maximum power increase observed at a resonator length detuning of zero was approximately a factor of 2.4 for one scan direction and smaller for the other scan direction. The non ideal form of one scan direction could be caused by absorption of the radiation by water vapour during the measurement. For the small resonances, which were observed at the relative resonator length change of 0.5 and -0.5 free spectral ranges, a relative power increase of 1.2 was measured. This power increase is proportional to the increase which is shown by the Airy function for double passing the resonator $\left(r=0.5^{2}=0.25\right)$. The resonance, which is seeded at 0.5 and -0.5 free spectral ranges, has a very low finesse. Responsible for this low finesse is the double circulation of the light through the resonator, passing the output coupler twice. Due to this low finesse only a low spectral purity of this mode can be obtained by injection seeding. The gain is not high enough to suppress the competing modes of the simple resonator. The measured cell transmissions are also corresponding well to the exponential Airy functions. At the main resonance the cell transmission follows the exponential Airy function of the simple resonator. The cell transmission of the resonances observed at 0.5 and -0.5 free spectral ranges are well described by the exponential Airy function of the double passed resonator. This is the reason for calling this effect 2 nd order injection seeding. A slight shift (in the order of $1 / 20$ FSR) between the calculated and the measured position of the second order modes is observed (see Fig. 7). Possibly this results

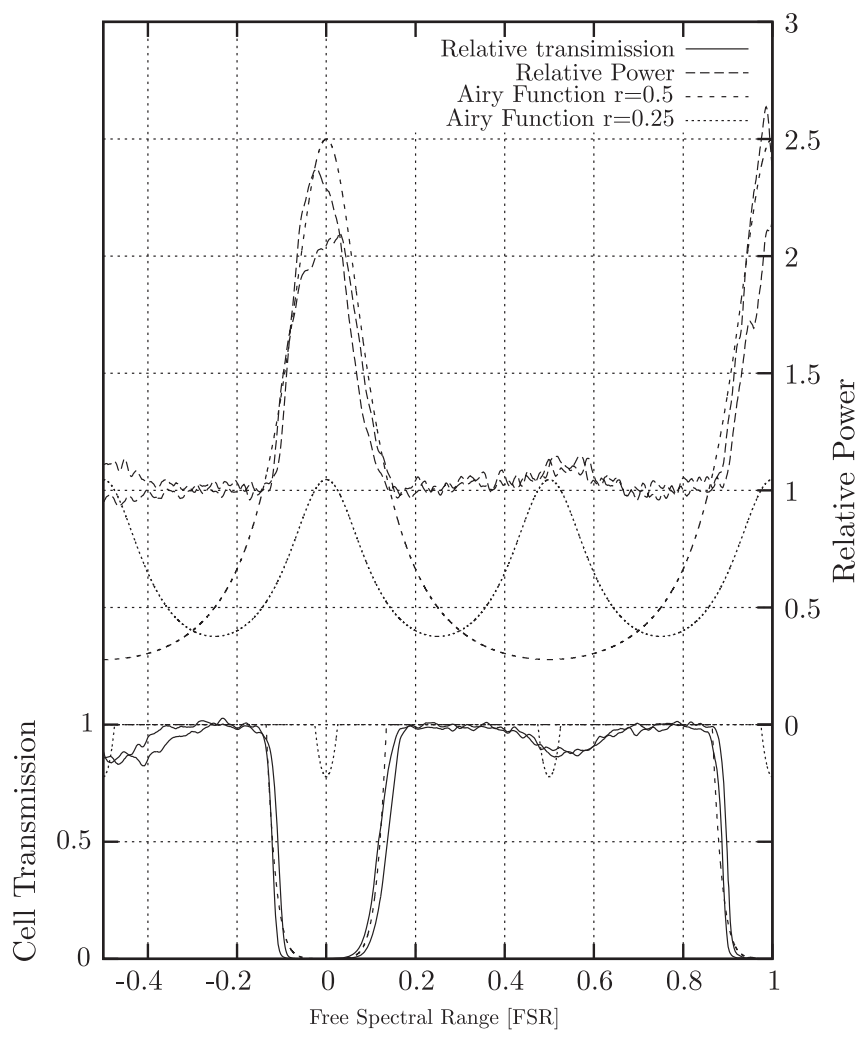

FIGURE 7 Observation of injection seeding of 2 nd order modes. The upper curves show the measured relative signal power of the OPO during one back and forth scan of the piezo. The lower curve shows the corresponding dependency of the cell transmission on the resonator length

from the different transversal mode structure of the second order modes.

The pump power range where the new feature is observed, can be estimated as followed: The maximum increase in power for the first order injection seeding is proportional to $\frac{1}{(1+r)^{2}}$ and the maximum power increase by second order injection seeding is proportional to $\frac{1}{\left(1+r^{2}\right)^{2}}$, the total maximum power increase needed to observe this phenomenon is expressed by the ratio of the two. For a total resonator loss of $50 \%$ this required power increase factor is 2.25 . Considering an OPO with a power curve like the one shown in Fig. 3 this increase is only observable for pump powers below 2.5 time above the threshold.

\section{$5 \quad$ Summary}

We described a four mirror configured injection seeded KTP OPO. A thorough investigation of the injection seeding parameters in dependence on the phase mismatch is given. We demonstrated injection seeding of a KTP OPO up to 13 -fold OPO threshold with a spectral purity of better than $99.7 \%$ for $\Delta k \approx 0$. The resonator length range $(\Delta l)$ where injection seeding was observed increases with increased pump power due to longitudinal line broadening by higher order transversal modes. However, we observed with increasing pump power a decrease of the acceptance range $(\Delta k)$ of the injection seeding caused by higher gain in the phase matched modes. 
As a result of the injection seeding experiments we discovered injection seeding between adjacent longitudinal resonator modes of the OPO at low pump powers. We interpret this feature as second order injection seeding.

\section{REFERENCES}

1 H. Flentje, A. Dörnbrack, G. Ehret, A. Fix, C. Kiemle, G. Poberaj, M. Wirth, J. Geophys. Res. 110, D03 115 (2005)

2 ESA, WALES - WAter vapor Lidar Experiment in Space, In: ESA SP1257(2)-Report for Assessment, ed. by P. Ingmann, A. Hélière (ESA Publication Division, ESTEC, Noordwijk, Netherlands, 2001)

3 R.T. White, Y. He, B.J. Orr, M. Kono, K.G.H. Baldwin, Opt. Express 12, 5655 (2004)

4 L.S. Rothman, D. Jacquemart, A. Barbe, D.C. Benner, M. Birk, L.R. Brown, M.R. Carleer, C. Chackerian, K. Chance, L.H. Coudert,
V. Dana, V.M. Devi, J.M. Flaud, R.R. Gamache, A. Goldman, J.M. Hartmann, K.W. Jucks, A.G. Maki, J.Y. Mandin, S.T. Massie, J. Orphal, A. Perrin, C.P. Rinsland, M.A.H. Smith, M.J. Tennyson, R.N. Tolchenov, R.A. Toth, J. Vander Auwera, P. Varanasi, G. Wagner, J. Quant. Spectrosc. Radiat. Transf. 96, 139 (2005)

5 P. Mahnke, H.H. Klingenberg, Appl. Phys. B 78, 171 (2004)

6 E.S. Cassedy, M. Jain, IEEE J. Quantum Electron. 15, 1291 (1979)

7 A. Fix, R. Wallenstein, J. Opt. Soc. Am. B 13, 2484 (1996)

8 A.V. Smith, SNLO Nonlinear Optics Code (Sandia Nat. Lab., Albuquerque, NM 87185-1423, 2006)

9 M.J.T. Milton, T.D. Gardiner, F. Molero, J. Galech, Opt. Commun. 142, 153 (1997)

10 R.L. Sutherland, Handbook of Nonlinear Optics (Marcel Dekker, New York, 1996)

11 A.E. Siegman, Lasers (University Science Books, Sausalito, CA, 1986)

12 A.E. Siegman, Appl. Opt. 1, 739 (1962) 